\title{
Proteaseaktivitätsbestimmung in Gewebshomogenaten und Extrakten mit Trinitrobenzolsulfonsäure
}

\author{
Von G. VALET \\ Aus dem Max-Planck-Institut für Biochemie (Direktor: Prof. Dr. A. Butenandt) Mïnchen
}

(Eingegangen am 8. Juli 1971)

Am Beispiel des Rattenleberhomogenats wird eine Methode zur Proteaseaktivitätsbestimmung von Gewebshomogenaten und Extrakten beschrieben, die auf der Reaktion der proteolytisch freigesetzten Aminogruppen der Spaltpeptide mit Trinitrobenzolsulfonsäure beruht. Die Quantifizierung der Aktivität in internationalen Enzymeinheiten U kann ohne Standard oder Eichkurve durchgeführt werden. Die Färbung ist bei Raumtemperatur für $12 \mathrm{Stdn}$ innerhalb $\pm 5 \%$ des Ausgangswertes stabil. Die Nachweisgrenze für Proteaseaktivität betrug bei Rattenleberhomogenat 0,56 mU. Sie kann mit gereinigten Enzympräparationen auf 0,19 mU erniedrigt werden. Als methodischer Fehler bei 10 Aktivitätsbestimmungen vom gleichen Rattenleberhomogenat bei $\mathrm{pH} 4$ crgab sich ein Variationskoeffizient von $4,9 \%$.

\section{The determination of protease activity in tissue homogenates and extracts with trinitrobenzenesulpbonic acid}

A method for the determination of protease activity is described, in which the free amino groups of the released peptides are reacted with trinitrobenzenesulphonic acid. The method was tested on rat liver homogenates and extracts. The enzyme activity can be quantified in international enzyme units without standards or a calibration curve. The colour is stable within $\pm 5 \%$ of the starting value at room temperature for $12 \mathrm{~h}$. The detection limit for protease activity in rat liver homogenate was $0.56 \mathrm{mU}$. This can be lowered to $0.19 \mathrm{mU}$ with purified enzyme preparations. 10 separate determinations of activity in the same rat liver homogenate at $\mathrm{pH} 4$ gave a variation coefficient of $4.9 \%$.

Von Lin, MeANs und FeENEY wurde eine Methode zur Messung der Proteaseaktivität gereinigter Enzyme angegeben, bei der die aus $\mathrm{N}, \mathrm{N}$-dimethyliertem Casein-, Hämoglobin- und Gelatinesubstrat freigesetzten $\alpha$ Aminogruppen der Spaltpeptide mit Trinitrobenzolsulfonsäure gefärbt werden (1). Sie hat gegenüber den Ansätzen nach Anson (2), nach KunITz (3) oder der Bestimmung des Ninhydrin-positiven Materials nach MOore und STEIN (4) den Vorteil, daB die gespaltenen Peptidbindungen quantitativ erfaßt werden.

Es wurde versucht, das Verfahren auf die Messung lysosomaler katheptischer Aktivitäten von Rattenleberund Rattenmilzhomogenaten und Extrakten anzuwenden. Wegen der geringen Proteaseaktivität in diesen Geweben wurden mg-Mengen der Enzympräparation zum Nachweis proteolytischer Aktivitäten benötigt. Infolge der Anfärbung freier Aminogruppen von Begleitproteinen der Enzympräparation ergaben sich trotz dimethylierten Hämoglobinsubstrats hohe photometrische Absorptionswerte in den Kontrollansätzen, die keine reproduzierbarte Aktivitätsmessung ermöglichten. Am Beispiel von Rattenleberhomogenat wird nachfolgend eine modifizierte Methode beschrieben, bei der die proteolytisch freigesetzten Aninogruppen der Spaltpeptide im Trichloressigsäureüberstand eines Ansatzes nach ANson (2) mit der von LiN, MEANS und FEENEY (1) verwendeten Trinitrobenzolsulfonsäure angefärbt werden.

\section{Methodik \\ Chemikalien}

Trinitrobenzolsulfonsäure von EGA-Chemie, 7924 Steinbeim und Serva, Heidelberg nach Reinigung über Norit A/Célite 535
(Serva) (5), Rinderhämoglobin nach Avson von Serva, die übrigen Chemikalien wurden in Analysenqualität verwendet.

Geräte

Zum Herstellen der Protease- und Trinitrophenylierungsansätze wurden Brand-Dispenser-Dosiergeräte (Brand, 6980 Wertheim) benützt. Die Inkubationen wurden in einem verschließbaren Wasserinkubator (Köttermann, 3165 Häningsen) durchgeführt. Der Trichloressigsäureüberstand wurde mit einer EppendorfMikroliter-Pipette entnommen. Die photometrischen Messungen wurden in einem Beckmann-DB-Zweistrahlphotometer bei $340 \mathrm{~nm}$ in Glasküvetten mit $1 \mathrm{~cm}$ Schichtdicke durchgeführt.

\section{Homogenat}

Die Homogenate wurden aus Lebern in Äthernarkose entbluteter weiblicher spezifiziert pathogenfreier Sprague-Dawley-Ratten mit einem Gewicht $z$ wischen $95-105 \mathrm{~g}$ hergestellt. Die Organe wurden sofort nach der Entnahme mit 4 Gewichtsteilen 0,25M Saccharose, $3 \mathrm{~mm} \mathrm{CaCl}$ bei $0^{\circ}$ in einem Potter-ElvehjemHomogenisator mit Teflonkolben $3 \mathrm{Min}$. bei $1500 \mathrm{U}$./Min. homogenisiert und die Homogenate bei $4^{\circ}$ aufbewahrt. Der Homogenatstickstoff wurde mit der Mikro-KJELDAHL-Methode nach Conway (6) bestimmt.

\section{Lösungen}

Als Inkubationspuffer für den Proteaseansatz wurde der Universalpuffer nach TeORELl und Stenhagen (7) in doppelter Konzentration verwendet. Zur Trinitrophenylierungsreaktion wurde ein $2 \mathrm{M}$ Boratpuffer aus $123,7 \mathrm{~g} \mathrm{H}_{3} \mathrm{BO}_{3}$ und $450 \mathrm{ml} 2 \mathrm{~N}$ $\mathrm{NaOH}$ Lösung je Liter Puffer hergestellt und bei etwa $60^{\circ}$ aufbewahrt. Hämoglobin $5 \mathrm{mg} / \mathrm{ml}$ und Trinitrobenzolsulfonsäure $1 \mathrm{mg} / \mathrm{ml}$ wurden zu jedem Versuch neu gelöst. Trichloressigsäure wurde als $1,2 \mathrm{M}$ Lösung verwendet.

\section{Proteaseansatz}

Folgende Ansätze wurden im Eisbad hergestellt (siehe Tabelle). Alle Ansätzc wurden $30 \mathrm{Min}$. bei $37^{\circ}$ im Wasserbad inkubiert mit Ausnahme des ,Nicht inkubierten Enzymansatzes', der bei $0^{\circ}$ gehalten wurde. Anschließend wurde das Protein aller Proben 


\begin{tabular}{|c|c|c|c|c|c|c|}
\hline Ansatz & $\underset{(\mathrm{ml})}{\text { Volumen }}$ & Bestandteile & $\begin{array}{c}\text { Konzentration } \\
\text { der } \\
\text { Bestandteile }\end{array}$ & $\begin{array}{r}\text { Konzentration de } \\
\text { im Ansatz, wenn } \\
\text { angegeben }\end{array}$ & $\begin{array}{l}r \text { Substanzen } \\
\text { nicht anders } \\
\text { in } \mathrm{mM}\end{array}$ & \\
\hline Proteolytischer Ansatz & $\begin{array}{l}1 \\
1 \\
1\end{array}$ & $\begin{array}{l}\text { Universalpuffer } \\
\text { pH } 2 \text { bis pH } 9 \\
\text { Hämoglobinlösung } \\
\text { Leberhomogenat }\end{array}$ & $\begin{array}{l}5 \mathrm{mg} / \mathrm{mi} \\
0,5 \mathrm{mgN} / \mathrm{ml}\end{array}$ & $\begin{array}{l}\mathrm{NaOH} \\
\text { Citronensäure } \\
\mathrm{H}_{2} \mathrm{PO}_{4} \\
\mathrm{H}_{3} \mathrm{BO}_{3} \\
\mathrm{HCl} \text { bei pH } 2 \\
\text { bei pH } 9 \\
\text { Hämoglobin } \\
\text { Leberhomogenat }\end{array}$ & $\begin{array}{r}45,7 \\
4,5 \\
6,9 \\
7,3 \\
48,8 \\
15,7 \\
1,67 \mathrm{mg} / \mathrm{ml} \\
0,167 \mathrm{mgN} / \mathrm{ml}\end{array}$ & - \\
\hline Inkubierter Enzymansatz & $\begin{array}{l}1 \\
2\end{array}$ & $\begin{array}{l}\text { Universalpuffer } \\
\text { pH } 2 \text { bis pH } 9 \\
\text { Leberhomogenat }\end{array}$ & $0,5 \mathrm{mgN} / \mathrm{ml}$ & $\begin{array}{l}\text { Puffersalze wie oben } \\
\text { Hämoglobin } \\
\text { Leberhomogenat }\end{array}$ & $\begin{array}{l}\text { angegeben } \\
\frac{0,333 \mathrm{mgN} / \mathrm{nil}}{}\end{array}$ & \\
\hline $\begin{array}{l}\text { Nicht inkubierter } \\
\text { Enzymansatz }\end{array}$ & $\begin{array}{l}1 \\
2\end{array}$ & $\begin{array}{l}\text { Universalpuffer } \\
\text { pH } 2 \text { und pH } 9 \\
\text { Leberhomogenat }\end{array}$ & $0,5 \mathrm{mgN} / \mathrm{ml}$ & $\begin{array}{l}\text { Puffersalze wie oben a } \\
\text { Hämoglobin } \\
\text { Leberhomogenat }\end{array}$ & $\begin{array}{l}\text { angegeben } \\
0,333 \mathrm{mgN} / \mathrm{ml}\end{array}$ & \\
\hline Substratansatz & $\begin{array}{l}1 \\
2\end{array}$ & $\begin{array}{l}\text { Universalpuffer } \\
\text { pH } 2 \text { und pH } 9 \\
\text { Hämoglobinlösung }\end{array}$ & $5 \mathrm{mg} / \mathrm{ml}$ & $\begin{array}{l}\text { Puffersalze wie oben } \\
\text { Hämoglobin } \\
\text { Leberhomogenat }\end{array}$ & $\begin{array}{l}\text { angegeben } \\
3,33 \mathrm{mg} / \mathrm{ml}\end{array}$ & \\
\hline $\begin{array}{l}\text { Trinitrobenzolsulfonsäure- } \\
\text { ansatz }\end{array}$ & $\begin{array}{l}1 \\
2\end{array}$ & $\begin{array}{l}\text { Universalpuffer } \\
\text { pH } 5 \\
\text { Wasser }\end{array}$ & & $\begin{array}{l}\text { Puffersalze wie oben } \\
\text { Hämoglobin } \\
\text { Leberhomogenat }\end{array}$ & angegeben & \\
\hline
\end{tabular}

im Eisbad mit $1 \mathrm{ml}$ 1,2M Trichloressigsäure gefällt und nach $10 \mathrm{Min}$. Wartezeit $10 \mathrm{Min}$. lang bei $3200 \mathrm{~g}$ abzentrifugiert. Für die nachfolgende Trinitrophenylierungsteaktion wurde aus allen $\mathrm{An}$ sätzen jeweils $1 \mathrm{ml}$ Uberstand entnommen und damit wie folgt verfahren:

Trinitropbenylieringsansatz

\begin{tabular}{|c|c|c|}
\hline$\underset{(\mathrm{ml})}{\text { Volumen }}$ & Bestandteile & $\begin{array}{l}\text { Konzentration } \\
\text { der Lösungen }\end{array}$ \\
\hline \multirow[t]{2}{*}{$\begin{array}{l}1 \\
1 \\
1 \\
1\end{array}$} & $\begin{array}{l}\text { Trichloressigsäureüberstand } \\
\text { NaOH } \\
\text { Boratpuffer pH } 9 \\
\text { Trinitrobenzolsulfonsäurelösung }\end{array}$ & $\begin{array}{c}0,35 \mathrm{~N} \\
2 \mathrm{M} \\
1 \mathrm{mg} / \mathrm{ml}\end{array}$ \\
\hline & $\begin{array}{l}\text { Inkubation: } 1 \text { Std. bei } 37^{\circ} \mathrm{im} \\
\text { abgedunkelten Wasserbad }\end{array}$ & \\
\hline $\begin{array}{r}10 \\
1\end{array}$ & $\begin{array}{l}\text { Wasser } \\
\mathrm{HCl}\end{array}$ & $2 \mathrm{~N}$ \\
\hline
\end{tabular}

Aus den trinitrophenylierten Ansätzen wurde die Proteaseaktivität folgendermaßen bestimmt:

\section{Proteaseaktivitätsbestimmung}

Die Proteaseaktivität der Enzympräparation mit dem zugesetzten Substrat Hämoglobin wurde als externe Aktivität bezeichnet und als Absorptionsdifferenz zwischen ,Proteolytischem Ansatz und einer Mischung gleicher Volumina, inkubierten Enzym' und ,Substratansatzes' im Photometer bestimmt. Die Aktivität der Proteasen in der Enzympräparation gegen die Begleitproteine wurde als interne Aktivität bezeichnet und als Absorptionsdifferenz zwischen, inkubiertem " und ,nicht inkubiertem Enzymansatz' gemessen. Die Konzentration der nicht fällbaren freien Aminogruppen in der Enzympräparation als Maß für die Konzentration freier Aminosäuren und Peptide im Lebergewebe wurde durch Messen des ,nicht inkubierten Enzymansatzes' gegen den ,Trinitrobenzolsulfonsäureansatz ${ }^{c}$ bestimmt. Die externe und interne Proteaseaktivität der Enzympräparation wurde aus den gemessenen Absorptionsdifferenzen in Enzymeinheiten U/mg Stickstoff ausgerechnet, wobei 1 Einheit U derjenigen Enzymaktivität entspricht, die in einer Minute bei $37^{\circ} 1 \mu \mathrm{Mol}$ Aminogruppen freisetzt. Folgende Formel wurde dazu verwendet:

Proteaseaktivität $=\frac{\Delta \mathrm{E} \cdot \mathrm{b} \cdot 10^{6}}{\mathrm{~d} \cdot \varepsilon \cdot \mathrm{t} \cdot \mathrm{a} \cdot}(\mathrm{U} / \mathrm{mgN})$

Die nicht fällbaren freien Aminogruppen in der Enzympräparation wurden wie folgt berechnet:

Freie Aminogruppen $=\frac{\Delta \mathrm{E} \cdot \mathrm{b} \cdot 10^{6}}{\mathrm{~d} \cdot \varepsilon \cdot \mathrm{a} \cdot}(\mu \mathrm{Mol} / \mathrm{mg} \mathrm{N})$
$\Delta \mathrm{E}=$ Extinktionsdifferenz. zwischen $M \mathrm{e} ß-$ und Vergleichsansatz, $\lambda=340 \mathrm{~nm}$

$=$ Molarer Absorptionskoeffizient der trinitrophenylierten freien Aminogruppen $\varepsilon=13500\left(\mathrm{Mol}^{-1} \cdot \mathrm{cm}^{2}\right)$ bei $340 \mathrm{~nm}$.

$\mathrm{d} \quad=$ Küvettenschichtdicke $(\mathrm{cm}), \mathrm{d}=1 \mathrm{~cm}$.
$\mathrm{t} \quad$ Dauer der Proteasejnkubation (Min.), $\mathrm{t}=30 \mathrm{Min}$.

a Menge der Enzympräparation im Trinitrophenylierungsansatz in Form des Trichloressigsäureüberstandes (moN). $a=0,125 \mathrm{mgN}$ für die externe Aktivität.

$a=0,250 \mathrm{mgN}$ für die interne Aktivität und die freien Aminogruppen.

b = Volumen des Trinitrophenylierungsansatzes (1), b $=0,015 \mathrm{l}$.

Die Ninbyydrinfärbung wurde nach MOORE und STEIN (4) und die Färbung mit dem Pbenolreagenz nach LOWRY, ROSEBRoUgH, FARR und RaNDALL (8) durchgeführt. Zur Bestimmung der mittleren Länge der Spaltpeptide in Aminosäureresten pro Peptid wurden die Trichloressigsäureüberstände aller Ansätze nach Hirs, MOORE und STEIN (9) alkalisch hydrolysiert, und sodann trinitrophenyliert. Die photometrischen Absorptionsdifferenzen dieser Ansätze wurden wie unter „Proteaseaktivitätsbestimmung“ beschrieben gemessen und als hydrolysierte externe und interne Aktivität bezeichnet. Die mittlere Länge der Spaltpeptide wurde aus dem Quotienten von hydrolysierter zu unhydrolysierter externer und interner Aktivität ausgerechnet.

\section{Ergebnisse}

Die Abbildung 1 zeigt die $\mathrm{pH}$-abhängigen Proteaseaktivitätskurven von Rattenleberhomogenat mit Aktivitätsmaxima bei pH 3,3.

Zum Vergleich der verschiedenen Aktivitätsbestimmungsmethoden für Proteasen wurden von den Tri-

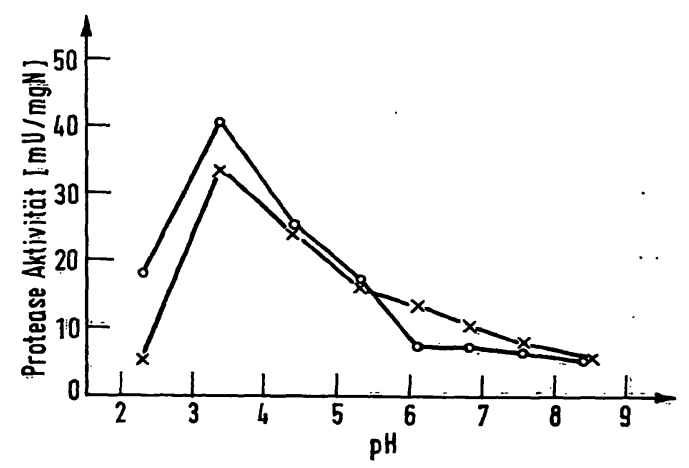

Abb. 1

Externe Proteaseaktivität von Rattènleberhomogenat gegen Rinderhämoglobin (o-o), sowie interne Proteaseaktivität $(\times-\times)$ gegen ansatz gemessen und blieb während der Inkubationszeit unverändert Die Aktivität ist in mU freigesetzte Aminogruppen pro mg Homogenatstickstoff angegeben 
chloressigsäureüberständen desselben Proteaseansatzes Aktivitätsbestimmungen nach ANsoN (2) und nach KunITZ (3), sowie Ninhydrin- (4) und Trinitrophenylierungsreaktionen durchgeführt. Die photometrischen Aktivitätswerte wurden auf $1 \mathrm{ml}$ Trichloressigsäureüberstand bezogen und sind zusammen mit den mittleren Längen der Spaltpeptide in Tabelle 1 aufgeführt.

Tab. 1

Vergleich der nach verschiedenen Verfahren gemessenen Proteaseaktivität von Rattenleberhomogenat bei $\mathrm{pH} 3$ pro ml Trichloressigsäureüberstand und der mittlereh Längen der Spaltpeptide für externe säureüberstand und der mittlereh Längen der

TBS $=$ Trinitrobenzolsulfonsäure $M P L=$ Mittlere Länge der Spaltpeptide (Anzahl der Aminosäurereste)

\begin{tabular}{lcc}
\hline Methode & $\begin{array}{c}\text { Externe } \\
\text { Aktivität } \\
\Delta \mathrm{E}\end{array}$ & $\begin{array}{c}\text { Interne } \\
\text { Aktivität } \\
\Delta \mathrm{E}\end{array}$ \\
\hline ANSON & 1,64 & 2,16 \\
KUNITz & 0,10 & 0,04 \\
Ninhydrin & 1,06 & 5,77 \\
TBS & 1,65 & $\mathbf{4 , 2 0}$ \\
\hline MPL & 4,0 & 1,6 \\
\hline
\end{tabular}

Die Methode nach KunItz (3) ist wenig empfindlich. Die Ninhydrinfärbung ist für die von interner Aktivität gebildeten kurzkettigen Peptide empfindlicher (5), für die durch externe Aktivität entstehenden längerkettigen Peptide unempfindlicher als die Trinitrophenylierungsreaktion. Der Ansatz nach ANsoN (2) zeigt die umgekehrte Tendenz. Das entspricht.insofern der Erwartung, als äquimolare Peptidmengen zunehmender Kettenlänge durch Ninhydrinreagenz schwächer (9), durch Lowry-Reagenz intensiver gefärbt werden (8). Die Trinitrobenzolsulfonsäurefärbung ergibt die einheitlichsten Aktivitätswerte, weil die molaren Absorptionskoeffizienten trinitrophenylierter $\alpha$ - und $\varepsilon$-Aminogruppen von Peptiden von deren Kettenlänge weitgehend unabhängig sind (10). Die Trinitrobenzolsulfonsäurefärbung ist bei Raumtemperatur mit einer Abweichung von maximal $\pm 5 \%$ des Ausgangswertes für mindestens $12 \mathrm{Stdn}$. stabil, während für die Lowry- und Ninhydrinfärbung nach 12 Stdn. nur noch $80 \%$ bzw. $60 \%$ der Ausgangswerte gemessen wurden.

Als methodischer Fehler für 10 Bestimmungen von Proteaseaktivität bei pH 4 und nicht fällbaren freien Aminogruppen vom gleichen Leberhomogenat wurden Variationskoeffizienten von $4,9 \%$ und $2,1 \%$ gefunden.

Die Nachweisgrenzen für Proteaseaktivität von Rattenleberhomogenat lag bei $0,56 \mathrm{mU}$, bei gereinigten Enzympräparationen können unter Weglassen der Wasserverdünnung der trinitrophenylierten Ansätze vor dem Ansäuern Proteaseaktivitäten bis $0,19 \mathrm{mU}$ nachgewiesen werden.

Herrn Prof. Dr. G. Runenstroth-Bauer bin ich für fortwährende Unterstützung sehr zu Dank verpflichtet.

Frl. Susan Mrtchell möchte ich für ausgezeichnete technische Assistenz danken.

\section{Literatur}

1. Lin, Y., G. E. Means und R. Feeney, J. biol. Chemistry 244 789 (1969). - 2. Anson, M. L., J. Gen. Phys. 2279 (1939). 3. Kunitz, J. Gen. Phys. 30291 (1946/47). - 4. Moore, S. und W. H. Stien, J. biol. Chem. 211907 (1954). - 5. Satake, K., T. Take, A. Matsuo, K. Tazaki und Y. Hiraga, J. Biochem, (Tokyo) 60, 654 (1966). - 6. Conway, E. J. und E. O'Malley, Biochem. J. 36, 655 (1942). - 7. Teorell, T. und E. Stenhagen,
Biochem. Zschr. 299, 419 (1938). - 8. Lowry, O. H., N. J. Rosebrough, A. L. Farr und R. J. Randall, J. biol. Chemistry 193, 265 (1951). - 9. Hirs, C. H. W., S. MOORE, W. H. Stein, J. biol. Chemistry 219, 623 (1956). - 10. SATAKE, K., T. OKUyama, M. Ofiashi und T. Shinoda, J. Biochem, (Tokyo) 47, 654 (1960).
Dr. G. Valet MPI f. Biochemie 8000 München 15 Goethestr. 31 